\title{
DARWIN - a next-generation liquid xenon observatory for dark matter and neutrino physics
}

\author{
Kevin Thieme ${ }^{a, *}$ on behalf of the DARWIN Collaboration \\ ${ }^{a}$ Department of Physics, University of Zurich, \\ Winterthurerstrasse 190, Zurich, Switzerland \\ E-mail: kevin.thieme@physik.uzh.ch
}

Benefiting from more than a decade of experience in WIMP searches with dual-phase xenon time projection chambers, the DARWIN (DARk matter WImp search with liquid xenoN) collaboration intends to build a next-generation detector involving 50 tonnes ( 40 tonnes active) of xenon. The primary goal of the observatory is to explore the entire experimentally accessible parameter space for WIMP masses above $5 \mathrm{GeV} / \mathrm{c}^{2}$ down to the irreducible neutrino floor. With its low energy threshold and ultra-low background level, DARWIN will be an excellent platform to search for various other rare interactions. These include the neutrinoless double beta decay of ${ }^{136} \mathrm{Xe}$, a high-precision measurement of the low-energy solar neutrino flux, as well as searches for solar axions and axion-like-particles. We present the detector concept, the sensitivity to the various science channels, and ongoing R\&D efforts.

$37^{\text {th }}$ International Cosmic Ray Conference (ICRC 2021)

July 12th - 23rd, 2021

Online - Berlin, Germany

\footnotetext{
${ }^{*}$ Presenter
} 


\section{Introduction}

Dual-phase noble gas time projection chambers (TPCs), in particular those operated with xenon, are the leading detector technology in the hunt for Weakly Interacting Massive Particles (WIMPs) in the mass range of a few $\mathrm{GeV} / \mathrm{c}^{2}$ to $\mathrm{TeV} / \mathrm{c}^{2}$ [1-3], cf. section 3.1, but are also successful in other rare event searches as in the case of the detection of two-neutrino double electron capture on ${ }^{124} \mathrm{Xe}$ [4]. In a dual-phase TPC, particle interactions in the liquid noble gas target are detected by a prompt scintillation light (S1) and a delayed electroluminescence signal (S2) caused by ionisation electrons that are drifted upwards and extracted into the gaseous phase by vertical electric fields. Both S1 and S2 signals are observed by photosensors sensitive to the vacuum ultraviolet (VUV) scintillation light. The drift time between S1 and S2 together with the horizontal S2 light distribution allow for a three-dimensional position reconstruction while the ratio of S1 and S2 is a discriminator for the type of interaction (electronic (ER) or nuclear recoil (NR)). The proposed DARWIN (DARk matter WImp search with liquid xenoN) observatory [5] builds upon the experience and know-how of the XENON Dark Matter Project [6] and can be regarded the ultimate evolution of this detector technology. The DARWIN project is a world-wide team effort of currently over 160 researchers from 33 institutions.

This contribution is structured as follows: We introduce the baseline detector design in section 2 and discuss its potential science reach and relevant backgrounds in section 3. The ongoing R\&D efforts towards the final detector design is covered in section 4 . We provide a summary of this contribution in section 5 .

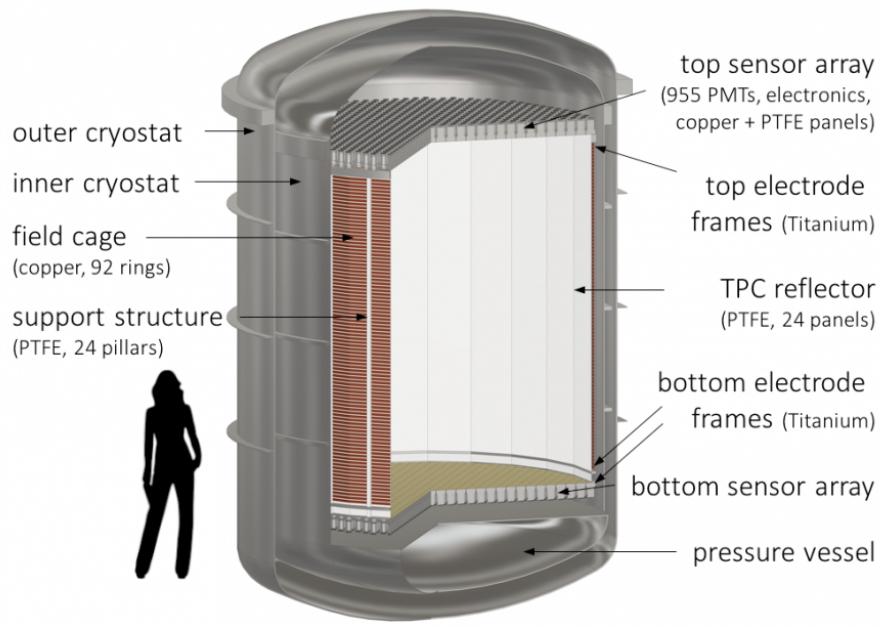

Figure 1: Possible realisation of the DARWIN TPC equipped with 3-inch PMTs inside the cryostat.

\section{Conceptual design}

In its baseline design presented in [5], DARWIN features a cylindrical TPC of both height and diameter of $2.6 \mathrm{~m}$ filled with an active xenon mass of $40 \mathrm{t}$ (50t total) and is surrounded by highly reflective PTFE walls, see figure 1 . The drift region is defined in the vertical direction by a negatively biased cathode and a gate at ground potential that provide a drift field of $O(0.1 \mathrm{kV} / \mathrm{cm})$. 
To ensure the uniformity of the electric field, equidistant field shaping rings from oxygen-free high-conductivity copper surround the detector volume. These are interconnected by a resistor chain to account for a linear potential change between the cathode and the gate. An extraction field of $O(1 \mathrm{kV} / \mathrm{cm})$ is created between the gate and the positively biased anode at the liquid/gas interface. Top and bottom photosensor arrays provide light and charge (also detected by light) readout and allow for position and energy reconstruction of interactions in the active volume. Past and present liquid xenon dark matter experiments use 3-inch Hamamatsu R11410 photomultiplier tubes (PMTs) as photosensors [6-9]. In the baseline scenario, the two photosensor arrays of a DARWIN-sized detector would be instrumented by $\sim 1800$ PMTs of this kind. In addition to this traditional solution, alternative novel photosensors are under study, see section 4 . The TPC is housed by a low-background double-walled cryostat which is surrounded by a neutron veto and a water Cherenkov detector to shield effectively from environmental radioactivity and from cosmic rays whose flux is highly suppressed by locating the facility in a deep underground laboratory. The sensitivity studies below consider the overburden of the Laboratori Nazionali del Gran Sasso (LNGS) as baseline.

\section{Sensitivity to the various science channels and relevant backgrounds}

Its high target mass, low energy threshold and ultra-low ER- and NR-induced background level will allow DARWIN to reach not only an unprecedented WIMP-sensitivity as primary goal but also to search for a variety of other rare processes that are discussed subsequently [5].

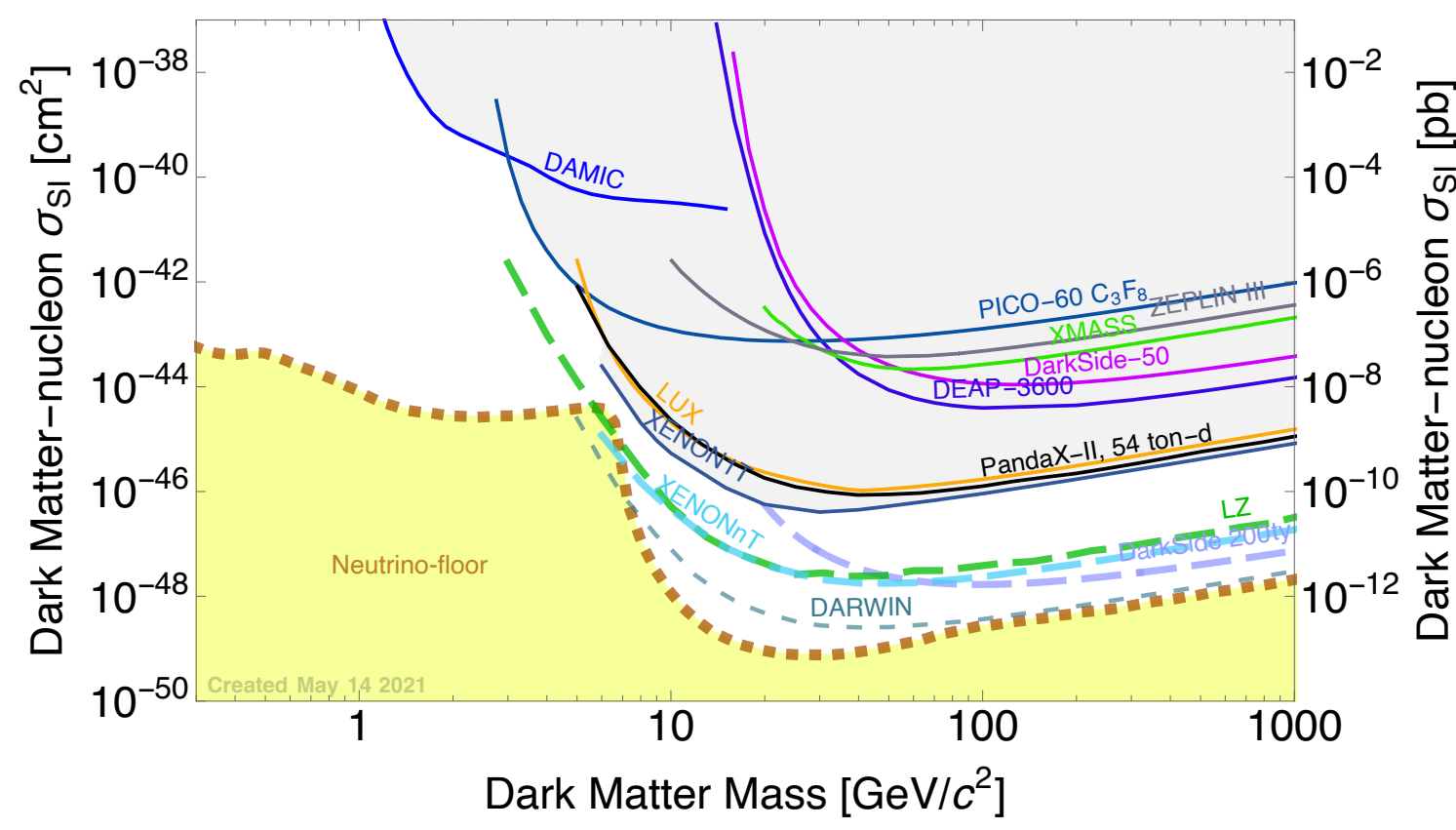

Figure 2: Past and present spin-independent limits on the WIMP-nucleon cross-section together with the projected sensitivities of the current generation experiments XENONnT and LZ [10]. DARWIN will push these sensitivities by another order of magnitude and ultimately reach the xenon neutrino floor. 


\subsection{WIMP dark matter}

As shown in [11], the projected spin-independent WIMP sensitivity of DARWIN for a $200 \mathrm{t} \times \mathrm{y}$ exposure of a $30 \mathrm{t}$ fiducial volume reaches $2.5 \times 10^{-49} \mathrm{~cm}^{2}$ for WIMPs with a mass of $40 \mathrm{GeV} / \mathrm{c}^{2}$, see in figure 2. This estimate is based on a series of background assumptions. Among those are a low concentration of $0.1 \mathrm{ppt}^{\text {nat }} \mathrm{Kr}^{1}$ and $0.1 \mu \mathrm{Bq} / \mathrm{kg}{ }^{222} \mathrm{Rn}^{2}$ in the liquid target. Important other ER backgrounds to the WIMP search are $\gamma$-rays from detector and cryostat materials, low-energy ( $p p$, $\left.{ }^{7} \mathrm{Be}\right)$ solar neutrino scattering on electrons and the two-neutrino double beta decay $(2 v \beta \beta)$ of ${ }^{136} \mathrm{Xe}$. Cosmogenic or radiogenic neutrons and coherent elastic neutrino-nucleus scattering (CEvNS) with solar (mostly ${ }^{8} \mathrm{~B}$ ) and atmospheric neutrinos present relevant NR background sources, that all leave a WIMP-like signal in the detector. Representing an irreducible background, the latter ultimately limits the sensitivity of this detector technology. The other named backgrounds can be mitigated by cryogenic distillation, target purification, a careful material selection and screening, fiducialisation, a neutron veto, and a water Cherenkov detector and shielding. The quoted sensitivity relies on an assumed ER-background rejection level of $99.98 \%$ at an NR-acceptance of $30 \% 3$ achieved by a uniform and high light and charge yield. In addition, DARWIN will complement measurements of the high-luminosity LHC (14 TeV) for spin-dependent WIMP-nucleon interactions and even probe the phase space above $1 \mathrm{TeV}$ WIMP mass, beyond the reach of the LHC [11].
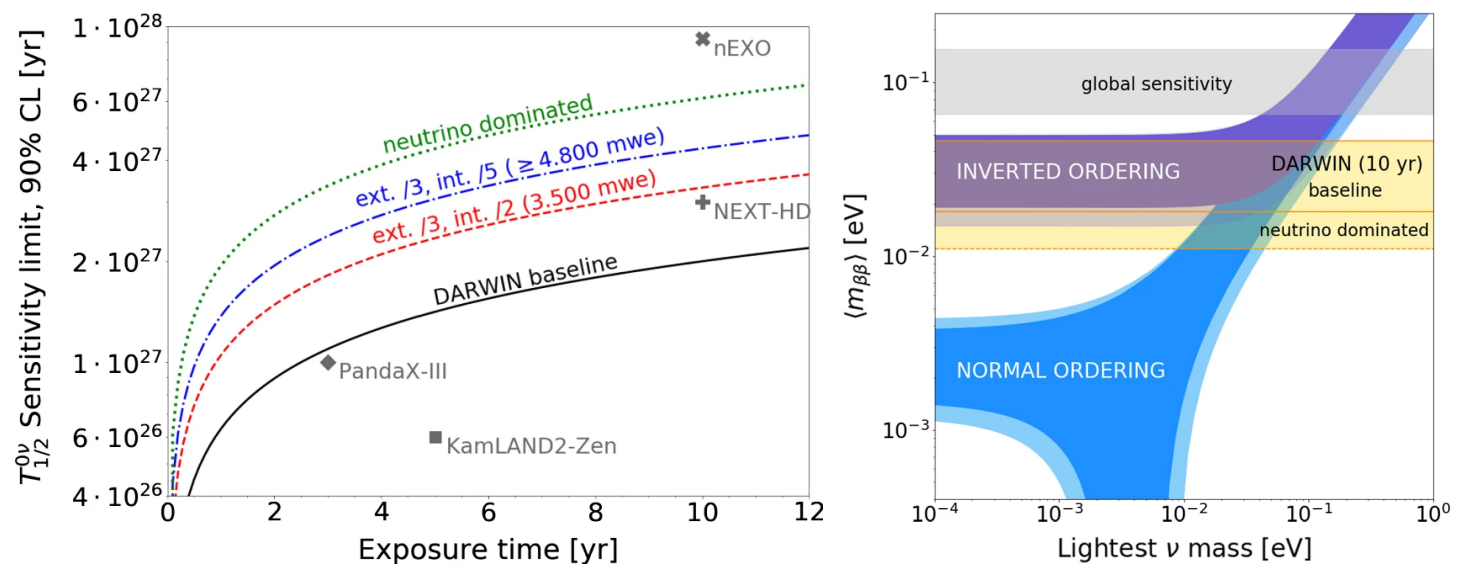

Figure 3: Left: Median half-life sensitivity of DARWIN at $90 \% \mathrm{CL}$ for an optimised $5 \mathrm{t}$ fiducial volume in comparison to future $0 v \beta \beta$ experiments. Shown are the results for the conservative baseline scenario and several more optimistic scenarios with reduced external (ext.) and internal (int.) backgrounds at different laboratory depths [14]. Right: Effective Majorana mass versus lightest neutrino mass. DARWIN covers the inverted ordering region of the neutrino Majorana mass for a $50 \mathrm{t} \times \mathrm{y}$ exposure [14].

\subsection{Neutrinoless double beta decay}

Natural xenon contains the isotope ${ }^{136} \mathrm{Xe}$ at an abundance of $8.9 \%$ which corresponds to an active mass of $3.5 \mathrm{t}$ inside DARWIN even without enrichment. This isotope is a candidate for the hypothetical neutrinoless double beta decay $(0 v \beta \beta)$ with an endpoint of the beta spectrum at

\footnotetext{
${ }^{1} \mathrm{~A}$ concentration of $0.03 \mathrm{ppt}$ has been achieved by the XENON collaboration in a cryogenic distillation test [12].

${ }^{2}$ This concentration is an order of magnitude lower than targeted by current experiments.

${ }^{3}$ This level of ER/NR discrimmination has been achieved in ZEPLIN-III [13].
} 
$Q_{\beta \beta}=2458 \mathrm{keV}$. This energy region is subject to a number of backgrounds. Besides those that are also relevant for the WIMP search like the uranium and thorium chains of the materials, ${ }^{222} \mathrm{Rn}$, ${ }^{8} \mathrm{~B}$ solar neutrinos and the standard $2 v \beta \beta$, this search is in particular influenced by $\beta^{-}$-decays from ${ }^{137} \mathrm{Xe}$ produced by capture of thermalised cosmogenic neutrons on ${ }^{136} \mathrm{Xe}$ inside the xenon target. For an optimised fiducial mass of $5 \mathrm{t}$ and incorporating background uncertainties, we calculated a $0 v \beta \beta$ half-life sensitivity of $T_{1 / 2}^{0 v}=2.4 \times 10^{27} \mathrm{y}(90 \% \mathrm{CL})$ in the baseline scenario with $3 \sigma$ discovery potential of $1.1 \times 10^{27} \mathrm{y}$ after an exposure of $10 \mathrm{y}$. This makes DARWIN competitive to dedicated $0 v \beta \beta$ near-future experiments, see figure 3 , left. The result corresponds to an effective Majorana mass of $m_{\beta \beta}=18-46 \mathrm{meV}$ and, hence, enables DARWIN to cover the inverted hierarchy mass region, see figure 3, right. A discussion of the Monte-Carlo background simulations, the sensitivity study and a comparison to near-future $0 v \beta \beta$ experiments can be found in [14].
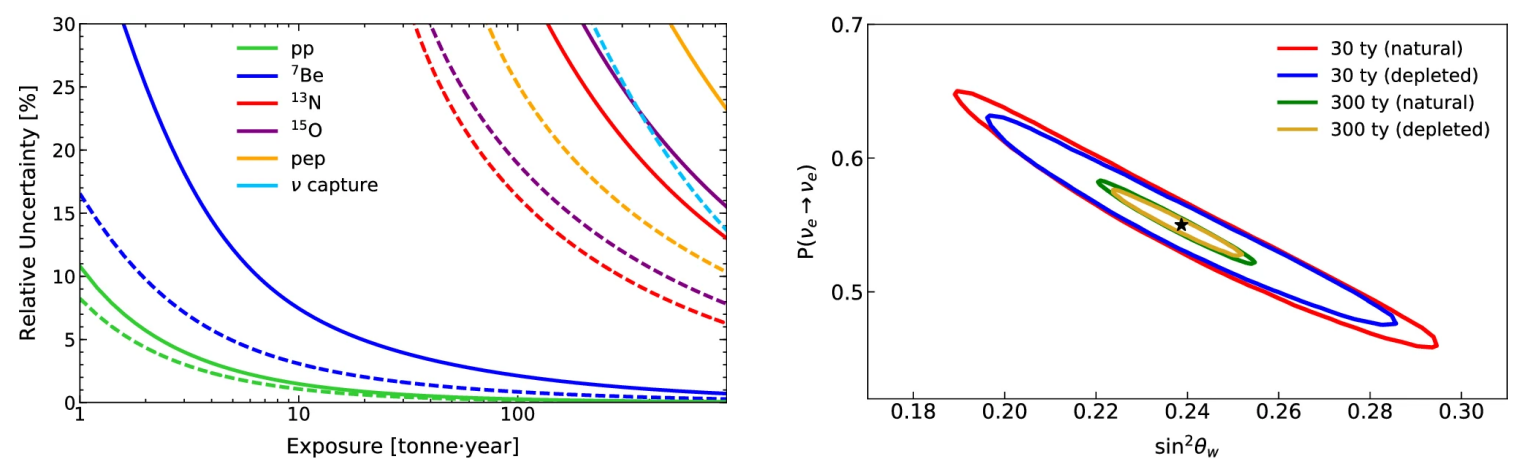

Figure 4: Left: Relative uncertainty on the flux of the solar neutrino components versus exposure [15]. Right: The $68 \%$ confidence regions on the weak mixing angle and the electron neutrino survival probability [15].

\subsection{Solar neutrinos}

While being a background to the aforementioned studies, elastic scattering of solar neutrinos off target electrons is an interesting science channel on its own [16]. Our detailed study on the matter can be found in [15]. Besides the background from the uranium and thorium chains in the detector and cryostat materials [14], the most significant backgrounds for the detection of lowenergy solar neutrinos come from internal ${ }^{222} \mathrm{Rn}$. Our study assumes a concentration of $2 \mathrm{ppq}$ ${ }^{\text {nat }} \mathrm{Kr}^{4}$ and $0.1 \mu \mathrm{Bq} / \mathrm{kg}{ }^{222} \mathrm{Rn}$ in the target. Furthermore, the double beta decay of ${ }^{136} \mathrm{Xe}$ presents a background which can be mitigated by depletion to the disadvantage of the $0 v \beta \beta$ prospects.

DARWIN can provide a high-precision measurement of the solar neutrino flux. In particular, with a $30 \mathrm{t}$ fiducial volume it would reach the $10 \%(2.7 \%)$ precision on the $p p\left({ }^{7} \mathrm{Be}\right)$ neutrino flux currently set by Borexino [17] with $1 \mathrm{t} \times \mathrm{y}(60 \mathrm{t} \times \mathrm{y})$ exposure and reach a precision of $0.15 \%$ $(1 \%)$ at $300 \mathrm{t} \times \mathrm{y}$, see figure 4 , left. These high-precision measurements would allow DARWIN to constrain the uncertainties of the neutrino-inferred solar luminosity to $0.2 \%$ and to determine the electroweak mixing angle $\sin ^{2} \theta_{W}$ and the electron-type neutrino survival probability $P_{v_{e} \rightarrow v_{e}}$ at yet unprobed low energies $(<200 \mathrm{keV})$ with a precision of $5.1 \%$ and $4.0 \%$ (for a natural xenon target), respectively (see figure 4 , right). The ${ }^{13} \mathrm{~N}$ and ${ }^{15} \mathrm{O}$ neutrinos are within reach as well. Sensitivity to

\footnotetext{
${ }^{4}$ This concentration is an order of magnitude lower than currently achieved [12]. However, even at the current level, it has only little effect on flux, electroweak mixing angle and electron neutrino survival probability measurements.
} 
the pep component and of the neutrino capture process on ${ }^{131} \mathrm{Xe}$, however, requires depletion from ${ }^{136} \mathrm{Xe}$ of the xenon target. Moreover, depletion would allow DARWIN to probe the metallicity of the Sun and help to distinguish high- and low-metallicity models up to the theoretical uncertainties.

\subsection{Other}

DARWIN is a unique platform to study various other channels. Among those are searches for solar axions, and for galactic axion-like particles and dark photons as light dark matter candidates [5]. A respective new sensitivity study is currently being performed. Other neutrino channels include the detection of $\mathrm{CE} v \mathrm{NS}$ with solar ${ }^{8} \mathrm{~B}$ neutrinos and neutrinos from galactic supernovae [18].
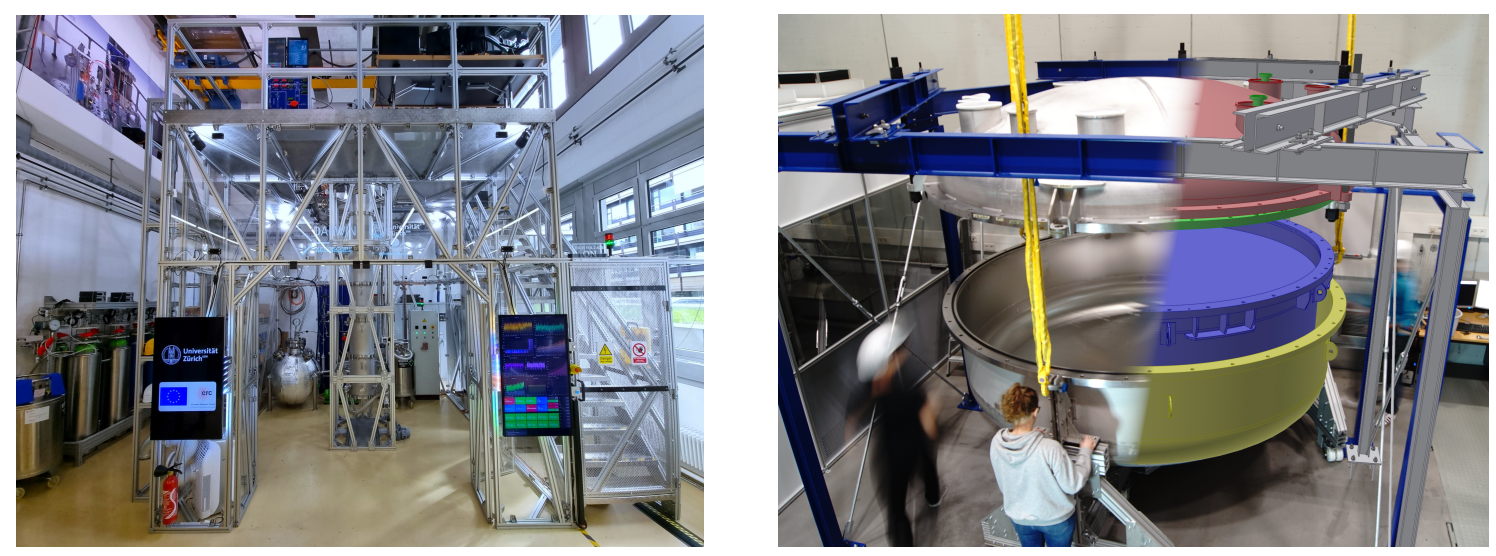

Figure 5: Full-scale DARWIN demonstrators. Left: Xenoscope at the University of Zurich [19]. Right: Pancake at the University of Freiburg.

\section{Research and development efforts}

To build a DARWIN-sized detector and meet the discussed requirements, a series of R\&D challenges have to be addressed and various key technologies to be tested. Two full-scale DARWIN demonstrators have been constructed to prove the working principle of the detector technology at these scales, and to test hardware and instrumentation under DARWIN-like conditions. Xenoscope at the University of Zurich is a full-height facility that was built to demonstrate the electron drift over a distance of $2.6 \mathrm{~m}$ and to address the liquid xenon purity requirements [19], see figure 5, left. Other objectives of Xenoscope are the high-voltage feed to the cathode as well as measurements of electron cloud diffusion and optical properties of xenon. Pancake at the University of Freiburg is a full-diameter facility with the primary goal of studying the mechanical and electrical behaviour of the electrodes and testing detector materials under cryogenic conditions to address e.g. uniform S2 amplification and shrinkage of the detector components.

Considerations on the detector design include, but are not limited to, the development of single phase liquid detectors, hermetic TPCs [20] and novel radon mitigation techniques. A number of alternative photosensors and readout schemes to the above mentioned 3-inch PMTs are under study or being developed. Among those are Silicon Photomultipliers (SiPMs) [21-24], Digital SiPMs, hybrid photosensors like ABALONE [25] and Vacuum Silicon Photomultiplier Tubes [26], but also other PMT models and novel ideas like bubble-assisted liquid hole multipliers [27] are considered. 


\section{Summary}

The DARWIN observatory is a proposed next-generation dark matter experiment that will not only explore the WIMP phase space above $5 \mathrm{GeV} / \mathrm{c}^{2}$ down to the irreducible neutrino floor but also be an excellent platform for other rare event searches. Among those are the neutrinoless double beta decay, solar neutrinos and neutrinos from supernovae, solar axions and galactic axion-like particles.

\section{References}

[1] XENON collaboration, Dark Matter Search Results from a One Ton-Year Exposure of XENON1T, Phys. Rev. Lett. 121 (2018) 111302 [1805.12562].

[2] LUX collaboration, Results from a search for dark matter in the complete LUX exposure, Phys. Rev. Lett. 118 (2017) 021303 [1608.07648].

[3] PandaX collaboration, Dark Matter Results From 54-Ton-Day Exposure of PandaX-II Experiment, Phys. Rev. Lett. 119 (2017) 181302 [1708.06917].

[4] XENON collaboration, Observation of two-neutrino double electron capture in ${ }^{124}$ Xe with XENONIT, Nature 568 (2019) 532 [1904.11002].

[5] DARWIN collaboration, DARWIN: towards the ultimate dark matter detector, JCAP 11 (2016) 017 [1606.07001].

[6] XENON collaboration, The XENONIT Dark Matter Experiment, Eur. Phys. J. C 77 (2017) $881[1708.07051]$.

[7] B.J. Mount et al., LUX-ZEPLIN (LZ) Technical Design Report, 1703.09144.

[8] PandaX collaboration, PandaX: A Liquid Xenon Dark Matter Experiment at CJPL, Sci. China Phys. Mech. Astron. 57 (2014) 1476 [1405 . 2882].

[9] XENONNT collaboration, Improved quality tests of R11410-21 photomultiplier tubes for the XENONnT experiment, 2104.15051.

[10] Dark Matter Limit Plotter v.5.16 by T. Saab and E. Figueroa. https://supercdms.slac.stanford.edu/dark-matter-limit-plotter.

[11] M. Schumann et al., Dark matter sensitivity of multi-ton liquid xenon detectors, JCAP 1510 (2015) 016 [1506.08309].

[12] XENON collaboration, Removing krypton from xenon by cryogenic distillation to the ppq level, Eur. Phys. J. C77 (2017) 275 [1612 . 04284].

[13] V.N. Lebedenko et al., Result from the First Science Run of the ZEPLIN-III Dark Matter Search Experiment, Phys. Rev. D 80 (2009) 052010 [0812 . 1150].

[14] DARWIN collaboration, Sensitivity of the DARWIN observatory to the neutrinoless double beta decay of ${ }^{136}$ Xe, Eur. Phys. J. C 80 (2020) 808 [2003 . 13407]. 
[15] DARWIN collaboration, Solar neutrino detection sensitivity in DARWIN via electron scattering, Eur. Phys. J. C 80 (2020) 1133 [2006.03114].

[16] L. Baudis et al., Neutrino physics with multi-ton scale liquid xenon detectors, JCAP 01 (2014) 044 [1309.7024].

[17] BOREXINO collaboration, Comprehensive measurement of pp-chain solar neutrinos, Nature 562 (2018) 505.

[18] R.F. Lang, C. McCabe, S. Reichard, M. Selvi and I. Tamborra, Supernova neutrino physics with xenon dark matter detectors: A timely perspective, Phys. Rev. D 94 (2016) 103009 [1606.09243].

[19] L. Baudis, Y. Biondi, M. Galloway, F. Girard, A. Manfredini, N. McFadden, R. Peres, P. Sanchez-Lucas and K. Thieme, Xenoscope - a full-scale vertical demonstrator for the DARWIN observatory, 2105.13829.

[20] K. Sato, M. Yamashita, K. Ichimura, Y. Itow, S. Kazama, S. Moriyama, K. Ozaki, T. Suzuki and R. Yamazaki, Development of a dual-phase xenon TPC with a quartz chamber for direct dark matter searches, PTEP 2020 (2020) 113H02 [1910. 13831].

[21] L. Baudis, M. Galloway, A. Kish, C. Marentini and J. Wulf, Characterisation of Silicon Photomultipliers for Liquid Xenon Detectors, JINST 13 (2018) P10022 [1808 . 06827].

[22] F. Arneodo, M.L. Benabderrahmane, G. Bruno, V. Conicella, A. Di Giovanni, O. Fawwaz, M. Messina, A. Candela and G. Franchi, Cryogenic readout for multiple VUV4 Multi-Pixel Photon Counters in liquid xenon, Nucl. Instrum. Meth. A 893 (2018) 117 [1707. 08004].

[23] L. Baudis, Y. Biondi, M. Galloway, F. Girard, S. Hochrein, S. Reichard, P. Sanchez-Lucas, $\mathrm{K}$. Thieme and J. Wulf, The first dual-phase xenon TPC equipped with silicon photomultipliers and characterisation with ${ }^{37}$ Ar, Eur. Phys. J. C 80 (2020) 477 [2003.01731].

[24] K. Ozaki, S. Kazama, M. Yamashita, Y. Itow and S. Moriyama, Characterization of New Silicon Photomultipliers with Low Dark Noise at Low Temperature, JINST 16 (2021) P03014 [2007.13537].

[25] D. Ferenc, A. Chang and M.S. Ferenc, The Novel ABALONE Photosensor Technology: 4-Year Long Tests of Vacuum Integrity, Internal Pumping and Afterpulsing, 1703.04546.

[26] G. Barbarino et al., A new generation photodetector for astroparticle physics: the VSiPMT, Astropart. Phys. 67 (2015) 18 [1407.2805].

[27] E. Erdal, L. Arazi, A. Breskin, S. Shchemelinin, A. Roy, A. Tesi, D. Vartsky and S. Bressler, Bubble-assisted Liquid Hole Multipliers in LXe and LAr: towards "local dual-phase TPCs", JINST 15 (2020) C04002 [1912 . 10698]. 